\title{
Tailored implementation of internet-based cognitive behavioural therapy in the multinational context of the ImpleMentAll project: a study protocol for a stepped wedge cluster randomized trial
}

Leah Bührmann ${ }^{1,2^{*}}$ D, Josien Schuurmans ${ }^{2,3}$, Jeroen Ruwaard ${ }^{2,3}$, Margot Fleuren ${ }^{1,2,4}$, Anne Etzelmüller ${ }^{1,2,5}$, Jordi Piera-Jiménez ${ }^{6}$, Tracy Finch, Tim Rapley ${ }^{8}$, Sebastian Potthoff ${ }^{9}$, Bruno Aouizerate ${ }^{10,11}$, Philip J. Batterham², Alison Calear ${ }^{12}$, Helen Christensen ${ }^{13}$, Claus Duedal Pedersen ${ }^{14}$, David Daniel Ebert ${ }^{1,5,15}$, Erik Van der Eycken ${ }^{16}$, Naim Fanaj ${ }^{17,18}$, Claire van Genugten ${ }^{2,3}$, Denise Hanssen ${ }^{19}$, Ulrich Hegerl ${ }^{20}$, Juliane Hug ${ }^{21}$, Annet Kleiboer ${ }^{1,2}$, Kim Mathiasen ${ }^{22}$, Carl May ${ }^{23}$, Sevim Mustafa ${ }^{17,24}$, Caroline Oehler ${ }^{25}$, Arlinda Cerga-Pashoja ${ }^{26}$, Catherine Pope ${ }^{27}$, Gentiana Qirjako ${ }^{28}$, Judith Rosmalen ${ }^{19}$, Ylenia Sacco ${ }^{29}$, Ludovic Samalin ${ }^{10,30}$, Mette Maria Skjøth ${ }^{14,31}$, Kristine Tarp ${ }^{32}$, Ingrid Titzler ${ }^{5,15}$, Enrico Zanalda ${ }^{29}$, Isabel Zbukvic ${ }^{3,34}$, Johannes H. Smit ${ }^{2,3}$, Heleen Riper ${ }^{1,2,3}$, Christiaan Vis ${ }^{1,2}$ and on behalf of the ImpleMentAll consortium

\section{Abstract}

Background: Internet-based Cognitive Behavioural Therapy (iCBT) is found effective in treating common mental disorders. However, the use of these interventions in routine care is limited. The international ImpleMentAll study is funded by the European Union's Horizon 2020 programme. It is concerned with studying and improving methods for implementing evidence-based iCBT services for common mental disorders in routine mental health care. A digitally accessible implementation toolkit (ItFits-toolkit) will be introduced to mental health care organizations with the aim to facilitate the ongoing implementation of iCBT services within local contexts. This study investigates the effectiveness of the ItFits-toolkit by comparing it to implementation-as-usual activities.

Methods: A stepped wedge cluster randomized controlled trial (SWT) design will be applied. Over a trial period of 30 months, the ItFits-toolkit will be introduced sequentially in twelve routine mental health care organizations in primary and specialist care across nine countries in Europe and Australia. Repeated measures are applied to assess change over time in the outcome variables. The effectiveness of the ItFits-toolkit will be assessed in terms of the (Continued on next page)

\footnotetext{
* Correspondence: l.buhrmann@vu.nl

'Department of Clinical, Neuro-, \& Developmental Psychology, Faculty of Behavioural and Movement Sciences, VU Amsterdam, Amsterdam, The Netherlands

${ }^{2}$ Amsterdam UMC, Vrije Universiteit Amsterdam, Psychiatry, Amsterdam Public Health Research Institute, Amsterdam, The Netherlands

Full list of author information is available at the end of the article
}

(c) The Author(s). 2020 Open Access This article is licensed under a Creative Commons Attribution 4.0 International License, which permits use, sharing, adaptation, distribution and reproduction in any medium or format, as long as you give appropriate credit to the original author(s) and the source, provide a link to the Creative Commons licence, and indicate if changes were made. The images or other third party material in this article are included in the article's Creative Commons licence, unless indicated otherwise in a credit line to the material. If material is not included in the article's Creative Commons licence and your intended use is not permitted by statutory regulation or exceeds the permitted use, you will need to obtain permission directly from the copyright holder. To view a copy of this licence, visit http://creativecommons.org/licenses/by/4.0/ The Creative Commons Public Domain Dedication waiver (http://creativecommons.org/publicdomain/zero/1.0/) applies to the data made available in this article, unless otherwise stated in a credit line to the data. 
(Continued from previous page)

degree of normalization of the use of the iCBT services. Several exploratory outcomes including uptake of the iCBT services will be measured to feed the interpretation of the primary outcome. Data will be collected via a centralized data collection system and analysed using generalized linear mixed modelling. A qualitative process evaluation of routine implementation activities and the use of the ItFits-toolkit will be conducted within this study.

Discussion: The ImpleMentAll study is a large-scale international research project designed to study the effectiveness of tailored implementation. Using a SWT design that allows to examine change over time, this study will investigate the effect of tailored implementation on the normalization of the use of iCBT services and their uptake. It will provide a better understanding of the process and methods of tailoring implementation strategies. If found effective, the ItFits-toolkit will be made accessible for mental health care service providers, to help them overcome their context-specific implementation challenges.

Trial registration: ClinicalTrials.gov NCT03652883. Retrospectively registered on 29 August 2018

Keywords: Tailored implementation, Normalization, Implementation strategies, Determinants of practice, eHealth, Mental health, Internet-delivered Cognitive Behavioural Therapy, iCBT, Stepped wedge trial design, SWT

\section{Background}

Common mental health disorders account for a large proportion of the global burden of disease [1]. Ample studies report on the clinical impact and other advantages of digital treatment for multiple mental disorders such as depression and anxiety, settings such as primary care or specialized care, and patient groups differing in diagnosis, severity levels, and comorbidities (e.g. [1-4]). Most frequently investigated are Internet-delivered Cognitive Behavioural Therapy (iCBT) services. Recent meta-analyses showed that self-guided iCBT is beneficial for patients with depressive symptoms $[5,6]$, and guided iCBT was found to have equivalent overall effects compared to face-to-face therapy for the treatment of psychiatric and somatic disorders [7]. Currently, research focusses on developing integrated treatment protocols blending face-to-face therapy with online treatment modules [8].

It is well recognized that evidence alone does not guarantee the effective use of an intervention in routine daily health care practice [9]. The implementation of iCBT services in routine care has varying degrees of success. Implementation is an intentional and planned process of normalizing (i.e. integrating and embedding) an innovation within an organization [10-12]. This process takes place at multiple organizational levels involving a wide range of stakeholders such as health care professionals, managerial staff, and/or patients. Cognitions and behaviours of clinicians and patients as well as organizational procedures are likely to remain in habitual patterns due to complex settings and working mechanisms [13]. Poor implementation contributes to the currently limited uptake numbers of evidence-based psychological treatments such as iCBT services in practice [14-19]. Few scientific studies have been published which systematically investigate and test implementation strategies and plans of such interventions. Efforts in the field primarily focussed on identifying and categorizing the factors hindering or enabling implementation processes. Folker and colleagues [20] described the scepticism of therapists and managers towards the use of iCBT, difficulties with the stable recruitment of patients, and problems with ensuring the long-term sustainability of the iCBT service. Other studies reported the general motivation and belief of professionals regarding the benefits of iCBT treatments [21, 22], but showed that lack of time, inadequate knowledge of the service, and the need to change habits were an impediment to the uptake of iCBT [22]. This is confirmed by a systematic review summarizing the determinants of practice regarding the implementation of Internet-delivered treatments [23]. Determinants of practice refer to any factor that hinders, enables, or facilitates the implementation process. The review highlighted 37 determinants to implementation on health care system, organizational, professional, and patient level showing that there is a multitude of barriers to overcome in order to implement iCBT successfully in routine practice. Depending on the context in which the implementation takes place and the nature of the service to be implemented, barriers differ in number and magnitude and might change over time. In that sense, every implementation setting is unique [24].

\section{Tailored implementation}

In order to overcome local barriers to implementation, suitable implementation strategies need to be applied. Implementation strategies refer to any kind of action aimed at accomplishing the integration of the innovation in practice (e.g. [18, 24-26]). Advances in the field of implementation science explore tailored approaches to develop implementation strategies. Tailored implementation strategies are defined as 'strategies to improve professional practice that are planned, taking account of prospectively identified determinants of practice' [27]. A 
Cochrane review of 32 studies showed that tailored implementation can be effective in comparison with nontailored implementation or no implementation interventions with small to moderate effect sizes when implementing practice guidelines in various health care settings [27]. They highlighted the importance of developing suitable methods to identify local determinants and to subsequently pair them to matching strategies. Following on from this work, the literature suggests that structured group methods involving stakeholders, such as brainstorming and focus groups, are successful methods to identify locally relevant determinants of practice [28]. A focus should be on the local contexts where the determinants emerge, as well as on the prioritization of certain determinants over others [28]. Besides differences in the type of innovation, technical infrastructures, and organizational processes, local contexts might also differ in their implementation culture and leadership. Organizational climates can be conductive to implementing evidence-based interventions, and leaders can employ various strategies to motivate and inspire others to implement innovative practices [29].

Building on previous research $[27,28,30]$, the ImpleMentAll project defines the concept of tailored implementation as follows: a prospective process involving systematic identification of determinants of practices within a local context, selection of implementation strategies appropriate to those determinants, the integration of these strategies into local work structures, and the actual application, evaluation, and potential further adaptation of the tailored implementation strategies (Fig. 1).
Thereby, tailoring is assumed to be a universal process applicable across health care contexts, settings, and care disciplines. In order to identify the most relevant determinants and the most suitable strategies to a local context, the identification process should be conducted using systematic methods [31]. The process of identification needs to involve a diverse group of stakeholder opinions to identify a variety of obstacles deemed important to the local situation [27]. As these considerations may substantially vary over time due to changes in internal and/or external circumstances of the organization, continuous tailoring throughout the implementation processes is of importance. Full details of the project-specific conceptualization of tailoring and its rationale will be discussed in a forthcoming publication.

\section{Objectives}

ImpleMentAll aims to evaluate the effectiveness of tailored implementation in integrating and embedding evidence-based $\mathrm{iCBT}$ services in routine mental health care for common mental disorders in adults. The projects conceptualization of tailored implementation is operationalized in an online platform, the ItFits-toolkit. Health care organizations, including primary and specialist mental health care, currently implement various types of iCBT services around the globe [32]. The ImpleMentAll consortium will use this natural laboratory to test whether the ItFits-toolkit will lead to favourable implementation outcomes compared to implementationas-usual in twelve ongoing implementation initiatives of $\mathrm{iCBT}$ in routine care in nine countries in Europe and

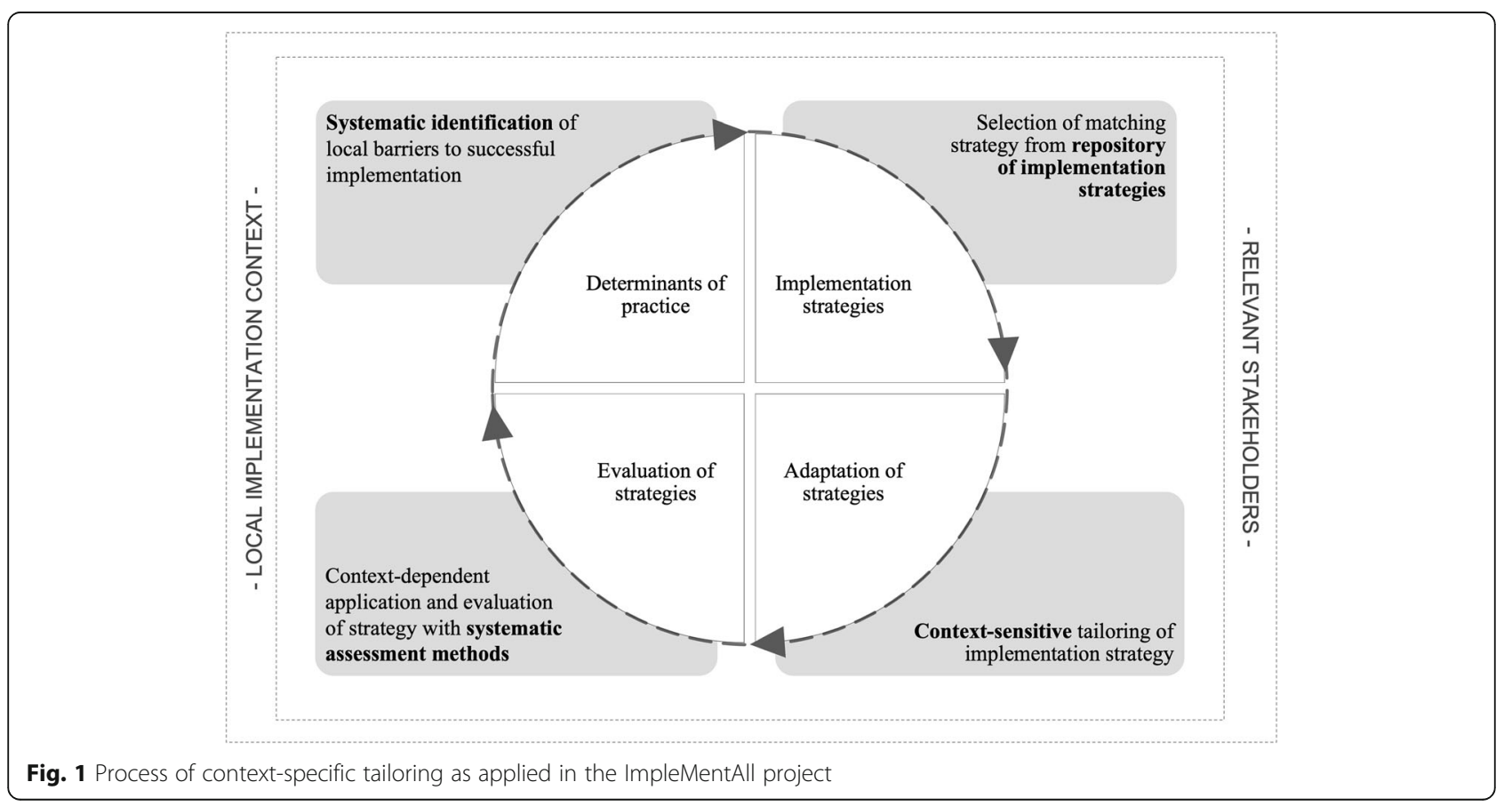


Australia. Successful implementation is hereby-primarily-defined as the normalization of the use of the iCBT services within the local settings. This paper presents the ImpleMentAll study protocol. The reporting follows the CONSORT extension for stepped wedge cluster randomized trials [33].

\section{Methods}

\section{Trial design}

A closed cohort stepped wedge cluster randomized controlled trial (SWT) design will be applied. Figure 2 schematically represents this design. Within a time period of 30 months, the ItFits-toolkit will be rolled out sequentially to twelve implementation sites (clusters). The twelve clusters are randomly allocated to six sequences (A-F) defining the different time points at which the clusters will cross over from the control condition (implementation-as-usual) to the experimental condition (ItFits-toolkit). As such, each cluster acts as both the control and experimental group over time. The successive cross-over points are scheduled every 3 months. The ItFits-toolkit will be used by each cluster for a minimum of 6 months $(b=$ minimal exposure period). During the 6-month exposure time, sites will receive technical support. The cohort will be encouraged to continue using the ItFits-toolkit after the minimal exposure period. Due to a potential intervention lag effect, it is expected that changes in the outcome measures become gradually visible in the data within and after the 6-month exposure period. As such, the effect is hypothesized to increase from no effect in the control condition (0) to a partial effect during the compulsory exposure period $(1 / 2)$ to a full and lasting effect after the 6-month exposure (1). Data will be collected 3-monthly (T0-T9) to strike a balance between the ability of the measurements capturing change over time and the measurement burden. A pre-rollout period of 6 months is chosen to obtain stable measures of implementation-as-usual activities in all clusters. That means the first three measurements (T0-T2) consist solely of implementation-asusual data and at $\mathrm{T} 2$ the first two clusters cross over to the experimental condition, followed by two clusters every 3 months.

\section{Study setting}

Twelve implementation sites from nine countries-Italy, Spain, Germany, France, The Netherlands, Denmark, Kosovo, Albania, and Australia-form the natural health care laboratory for this study. The health care systems across the implementation sites differ regarding the organization of mental health service delivery, including aim and type of iCBT services offered (treatment or
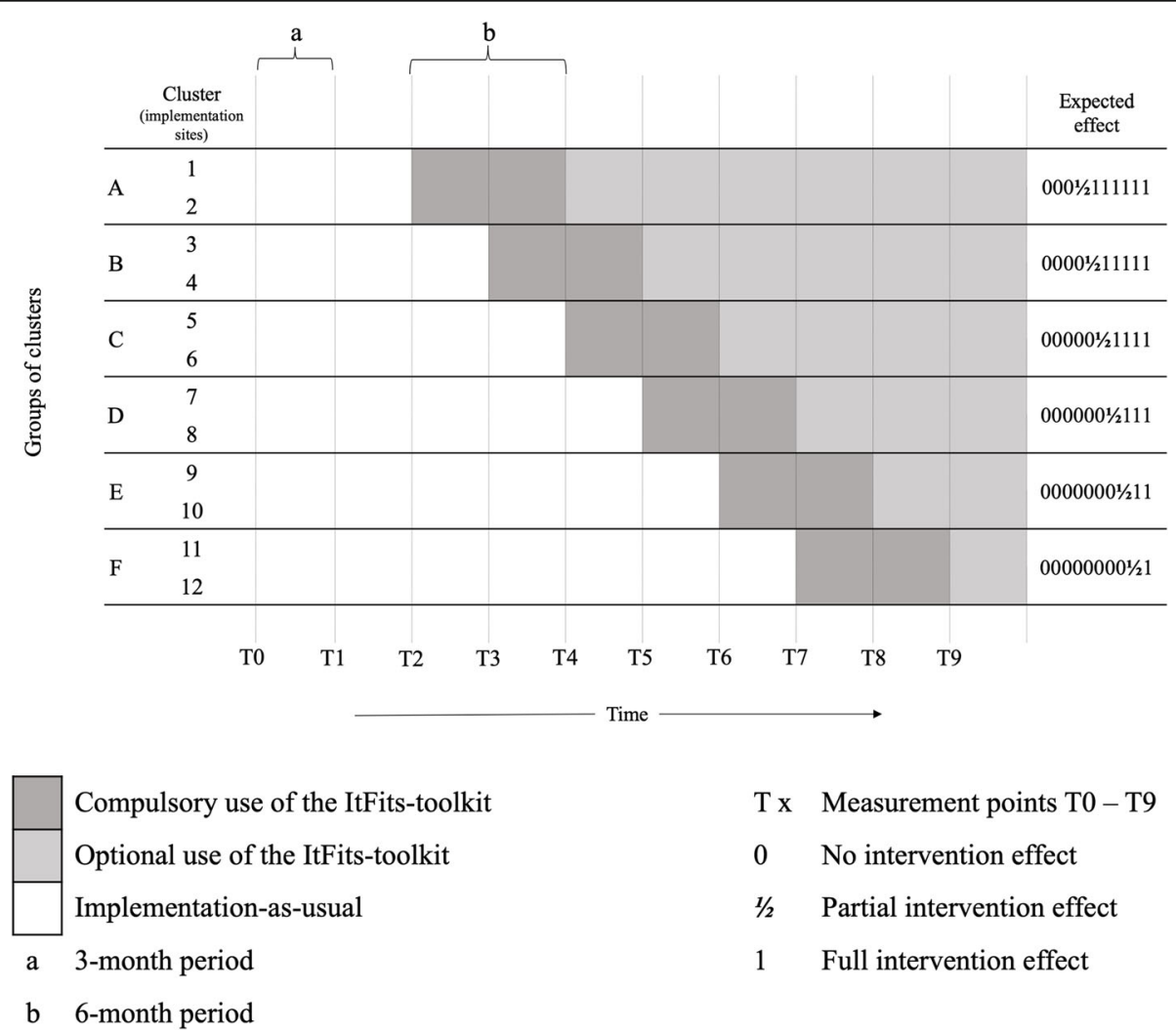

$\mathrm{T} \mathrm{x}$ Measurement points $\mathrm{T} 0-\mathrm{T} 9$

$0 \quad$ No intervention effect

$1 / 2$ Partial intervention effect

1 Full intervention effect

Fig. 2 Stepped wedge cluster randomized trial design for the ImpleMentAll project 
prevention, and self-help, guided, or blended format), clinical pathways, guidelines, procedures, and cultures, as well as financing and legislative. Within those care settings, the participating implementation sites' mental health services are located in community care, in primary or specialized care, or in a stepped-care model. Referral pathways include self-referral, as well as referral by GPs, psychologists, psychotherapists, or insurance companies.

All implementation sites have adopted and are implementing prevention or treatment services of mild to moderate depressive disorder, anxiety disorder, substance abuse, and medically unexplained symptoms or somatic symptom disorders. The iCBT services are based on the working mechanisms of Cognitive Behavioural Therapy covering four main components: psychoeducation, techniques invoking behavioural change, a cognitive component, and relapse prevention [34]. All services make use of Internet technology. However, the specific operationalization differs per service in response to the local requirements. Similarly, various guidance modalities are embedded in the iCBT services, ranging from self-help with minimal technological and administrative support, to therapist guided treatments, and blended approaches where online modules and face-toface therapy are integrated into one treatment protocol.
Patient pathways, clinical eligibility criteria for receiving the iCBT service, as well as stopping rules of participation follow local guidelines and procedures applicable in the implementation sites.

\section{Participants}

Following the SWT design reporting guidelines [33], participants are classified at two levels: (1) implementation sites (organizations) as cluster-level participants represented by individuals responsible for the local implementation work (implementers) and (2) staff within these sites as individual-level participants. The anticipated participant flow through the study is schematically depicted in Fig. 3.

\section{Organizational (cluster) level}

The implementation sites eligible to partake in the study are engaged in the implementation of $\mathrm{iCBT}$ at least 3 months prior to the baseline measurement. Each implementation site is responsible to recruit a sufficient number of staff participants (see the "Sample size and power estimates" section).

\section{Staff (individual) level}

Every individual involved in the delivery of the iCBT service within the participating implementation site is

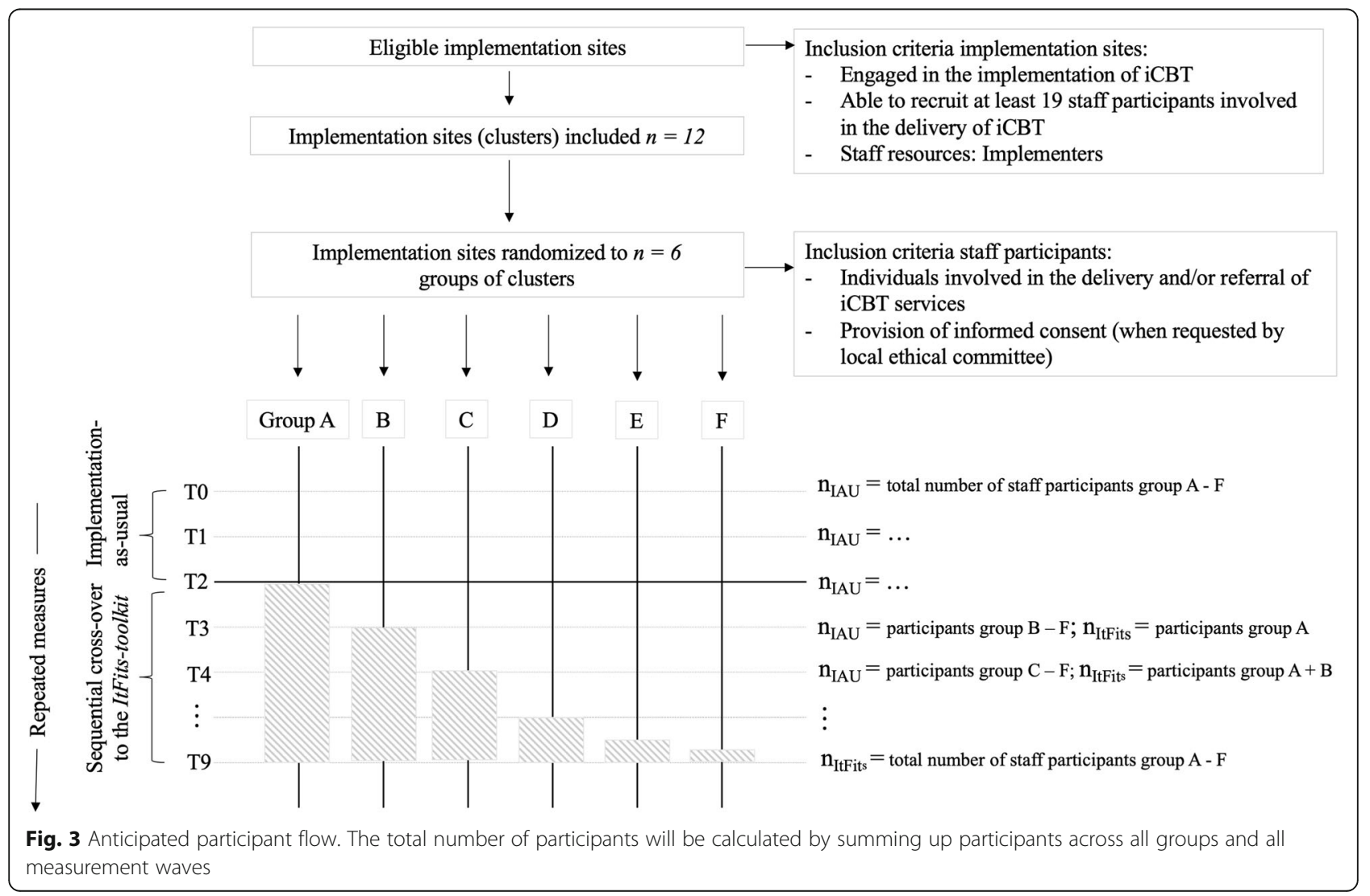


eligible to take part in the study. Staff participants can have different roles in the delivery of $\mathrm{ICBT}$ and include therapists, such as psychologists, psychiatrists, or mental health nurses; referrers such as GPs, pharmacists, community workers, health promotors, counselors, or case managers; administrators such as clerical workers or secretaries; ICT support staff, such as security officers, maintenance officers, or helpdesk staff; and managers of the organizations. Staff participants will give their informed consent in accordance with local and European directives for the protection of privacy, voluntary participation, and the right to withdraw from the study at any given time. Staff is excluded from participation when they are enrolled in the study as local implementers or when they are involved in any activities of the local or central trial management.

\section{Conditions}

\section{The experimental condition: the ItFits-toolkit}

The online self-help implementation toolkit 'ItFits' aims at supporting implementers in developing, applying, and monitoring implementation strategies that are adapted to local contexts to integrate and embed iCBT services in routine mental health care. The ItFits-toolkit has the potential to impact the implementation on various levels (e.g. at staff, patient, organizational, and policy level). Examples may include the adaptation of organizational workflows, personnel decisions, training and motivation, or modifications of the service delivery mode. The ItFitstoolkit is based on scientific output and theories in the field of implementation [30, 35, 36]. To ensure an appropriate balance of being theoretically informed whilst also practically orientated and accessible to non-academic users, the ItFits-toolkit has undergone rounds of conceptual and technical piloting, with user groups representing a range of relevant perspectives.

Within each implementation site in the ImpleMentAll study, a self-guided implementation core team (up to four staff members internally to the organization (implementers) represented by an implementation lead) will be established. These teams are likely to include therapists and other professionals involved in the delivery of the iCBT service, but may also include individuals from partner organizations where appropriate, for example, if they are invested stakeholders in the service (e.g. commissioners). The implementation core team will coordinate and work with the ItFits-toolkit. In four modules, concrete guidance on tailoring implementation strategies to local determinants of practices will be provided, applied, and evaluated. The four modules are (1) identifying and prioritizing implementation goals and determinants of practices, (2) matching up implementation determinants to strategies, (3) designing a plan for carrying out strategies in a local context, and (4) applying strategies and reviewing progress. In the last module, a decision will be made whether the implementation strategy will be stopped (in case of perceived success), continued, or redesigned. Figure 4 illustrates the workflow of the ItFits-toolkit. An overview of the main working components of the ItFits-toolkit is summarized in Table 1.

Within the four modules, the ItFits-toolkit employs a systematically guided but flexible step-by-step process, including stakeholder-based co-creation. The Normalization Process Theory (NPT) explains that successful integration and embedding are achieved through people (individuals and groups) working together [35]. Therefore, engagement and consultation of staff involved in the implementation work and in $\mathrm{ICBT}$ service delivery are a core feature of the toolkit. Stakeholders are individuals which either actively affect the iCBT service (e.g. service delivery staff, IT staff, managers) or are passively affected by the delivery of the iCBT service (e.g. patients). Implementers work through a three-step iterative process (see Fig. 5) in order to reach the best possible outcome for each module. The local implementation core team develops an initial plan or idea, which is discussed with and reviewed by the stakeholders for feedback in order to design a feasible plan that reflects the needs, priorities, and restraints in the local situation. Subsequently, the implementation core team finalizes the plan to accommodate stakeholders' feedback. For each module, different stakeholders might be consulted depending on the task at hand.

In order to engage with stakeholders throughout the process, a number of consensus techniques are recommended, including brainstorming, structured group discussions, individual and informal discussions, and surveying [28]. Surveying functionalities are embedded within the ItFits-toolkit to allow implementers to flexibly create dedicated online surveys which they can administer to relevant stakeholders via email. In addition, the toolkit allows implementers to upload and store notes, audio recordings, and other relevant materials, which document the decisions and progress made and can be used for reviewing purposes. Implementers are also actively working with evidence-informed materials, including literature on iCBT relevant determinants of practice $[23,37,38]$ and implementation strategies $[25,26,39]$, as well as guidelines to develop and structure the tailored implementation plan [40].

The commitment of study sites to participate in the trial and to use the toolkit according to the study protocol has been agreed in advance, and resources have been allocated to support their participation. Each participating site is required to work with the toolkit for at least 6 months and is instructed to strive to finish all four modules within this time period. The intensity of use 


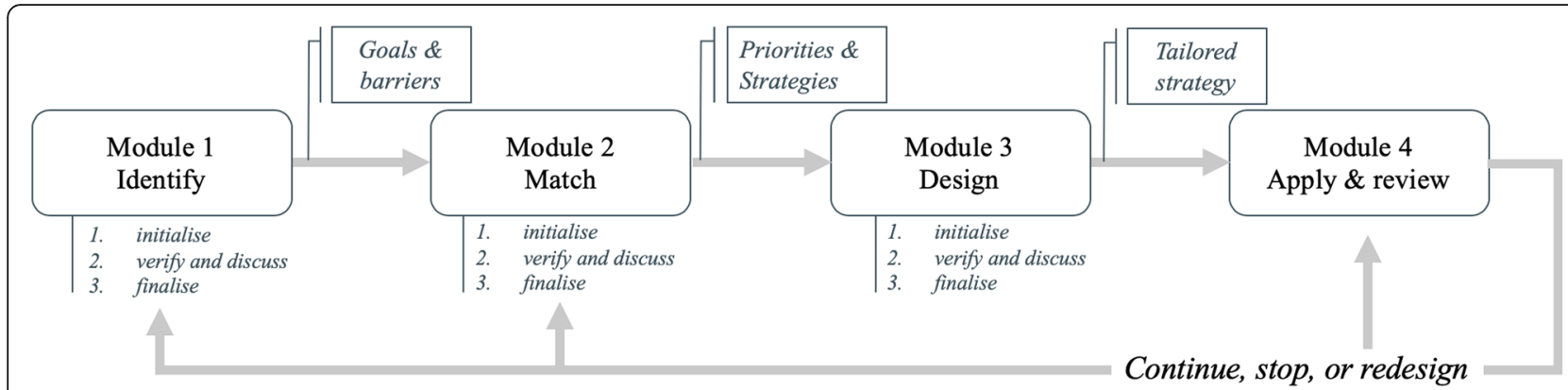

Fig. 4 Flowchart of the ItFits-toolkit

depends on the local context and needs. For the toolkit, a protocolized introductory training will be provided, and periodic support (in form of monthly support sessions and assistance on request) will be available, focusing solely on technical questions, such as login procedures, the use of tools, or the navigation through the platform, to ensure a smooth working process without interfering with the working components of the toolkit. Within the toolkit itself, there is access to written, audio, and video guidance to completing activities within the modules. The training and support will be provided by two members of the research team.

\section{The control condition: implementation-as-usual}

Implementation-as-usual functions as the control condition in testing the effectiveness of the ItFits-toolkit. It refers to any existing activities the implementation sites are engaged in to embed and integrate the local iCBT programme within routine care. Examples include the provision of training to iCBT service deliverers, educating staff and patients on the use of $\mathrm{iCBT}$, or media campaigns to raise awareness of $\mathrm{iCBT}$ services. The implementation sites started the processes of routinely implementing the $\mathrm{iCBT}$ services at least 3 months prior to baseline (T0) with the goal of continuously improving the utilization of the services.

\section{Outcomes}

\section{Primary outcome}

The effectiveness of the ItFits-toolkit will be expressed in the extent to which the ItFits-toolkit is able to increase the degree of normalization of the use of the iCBT

Table 1 Core working principles of the ItFits-toolkit

1) Flexible, systematic step-by-step workflow

2) Stakeholder-based co-creation to reach consensus

3) Tools to identify local barriers, consult stakeholders, and match to suitable strategies

4) Evidence-informed materials on barriers, strategies, and intervention planning services compared to usual implementation activities. The degree of normalization is the extent to which staff involved in service delivery and referral consider the iCBT service to be a normal part of their routine practice. It will be measured with the 20-item Normalization MeAsurement Development tool, short NoMAD [41, 42]. The NoMAD is a self-report instrument measuring the degree of normalization by focusing on four constructs as defined by the Normalization Process Theory [35]: coherence, cognitive participation, collective action, and reflexive monitoring. The NoMAD shows high internal consistency and has been validated in heterogeneous samples across languages and settings [42-44].

\section{Exploratory outcomes}

Service uptake Service uptake is defined in terms of the completion rate of the $\mathrm{iCBT}$ service, that is, the absolute

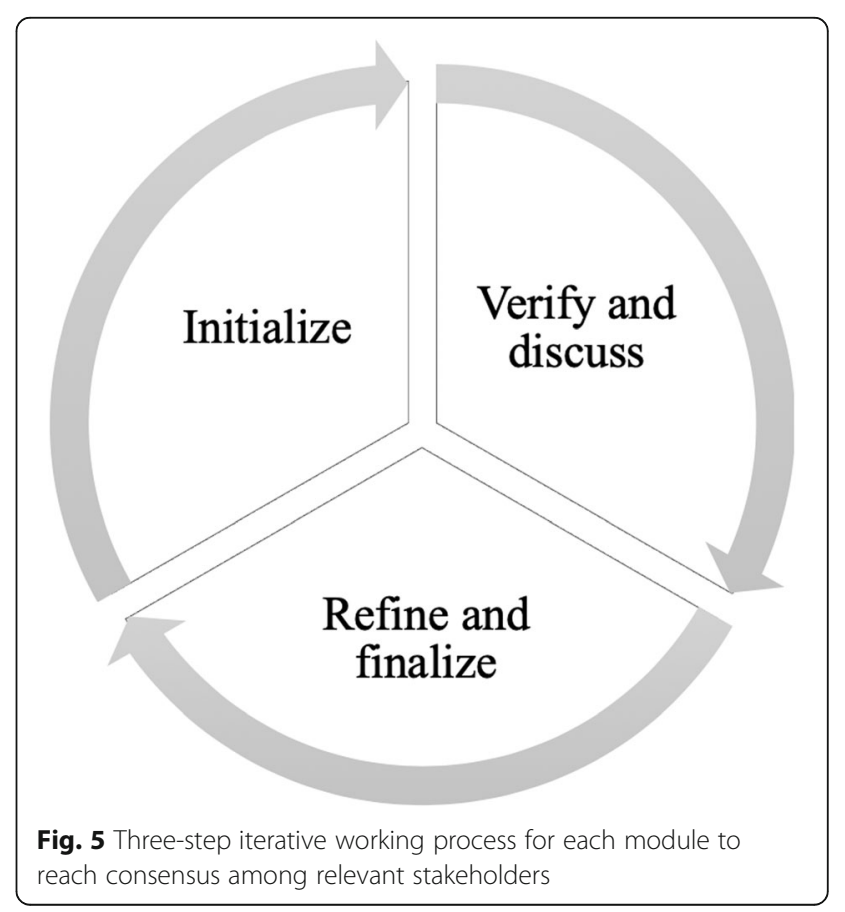


number of patients actually completing the trajectory of the iCBT service during the trial period. Each implementation site has their own protocolized or experiencebased operationalization of 'completion' according to the therapeutic principles and procedures of the local service being implemented. In addition to collecting data on completion rates, also data on referral rates will be collected to investigate the effect of the ItFits-toolkit on iCBT service uptake by staff, i.e. referral to the service.

Implementation costs Efficiency is defined as the ratios between implementation cost and service uptake, and implementation cost and degree of normalization. Within the ImpleMentAll study, implementation costs are defined as the sum of personnel costs, other direct costs, and indirect costs. Personnel costs are calculated by multiplying the working hours spent by the implementers on implementing the iCBT service by standardized hourly wage rates and a standardized country correction factor. Other direct costs include costs for consumables, equipment, and services purchased for the purpose of carrying out the implementation activities. Indirect costs are costs that cannot be attributed directly to the implementation work but are necessary to facilitate the implementation, such as office rent, office IT infrastructure, or administration. Indirect costs are calculated by taking $20 \%$ of the direct costs. Implementation costs (i.e. personnel costs, other direct costs, and indirect costs) will be assessed in the local implementation settings by monitoring the different cost components over time.

Exposure Exposure to the ItFits-toolkit will serve as a measure to determine if the measured change in outcomes can reliably be related to the use of the toolkit. Usage data will be automatically collected by system logs recording time stamped starting and stopping of ItFitsmodules (use) and a binary confirmation of the existence of output of the modules (result).

Satisfaction As the ItFits-toolkit is a newly developed tool, satisfaction of use will be assessed to establish to what extent the toolkit is able to fulfil implementers' needs and expectations in tailored implementation. Satisfaction will be assessed with the short version of the Client Satisfaction Questionnaire (CSQ-3) [45], which has good psychometric properties and has been tested in numerous studies and diverse samples [46, 47].

Usability Usability will be measured with the validated System Usability Scale (SUS) $[48,49]$. The instrument measures the perceived, local usability of the ItFits-toolkit-such as complexity of the tool, user experience, and perception of the technological realization-by the toolkit user.

Impact The perceived impact will be assessed to explore whether the implementation strategies developed by using the ItFits-toolkit are considered to be satisfactory in fulfilling the implementers' needs. The perceived impact will be measured using a visual analogue scale.

Organizational readiness Organizational readiness for implementing change $[50,51]$ is hypothesized to be a potential precursor or contextual factor for implementation success [52]. This concept will be assessed using the 'Organizational Readiness for Implementing Change' (ORIC) questionnaire [51], which focuses on the psychological and behavioural preparedness of members of an organization to implement organizational change [51]. Considering similarities in theoretical concepts, ORIC questionnaire data will also be used to explore its convergent validity with the NoMAD instrument.

Table 2 summarizes the primary and exploratory outcomes. All staff-level questionnaires have been translated and adapted into local languages using a standardized translation guide. The translated versions of the instruments are available for public use [53].

\section{Data collection}

Data will be collected on implementation sites and staff level through (1) a central data management system specifically built for the purpose of the study and (2) eventbased $\log$ files of the ItFits-toolkit. Online surveys will automatically be sent to participants via email with the request to fill in a specified measure. The measurement time points are pre-scheduled for 3-month intervals during the 30-month study period. Data on demographics are collected once, when a participant enters the study. Degree of normalization, uptake, implementation costs, and organizational readiness for change will be measured at 3-monthly intervals. Exposure data will be collected continuously, and data on satisfaction and usability will be recorded at the end of the exposure period (see Table 2). Depending on the participant's activity, reminders will be sent at regular intervals to ensure continuous completion of the questionnaires by each participant. The burden for study participants to provide the required data is kept to a minimum by using brief online questionnaires and automatically collected data.

\section{Sample size and power estimates}

This study has a fixed cluster sample size by design $(n=$ 12 implementation sites) based on availability and willingness of organizations engaged in implementation of iCBT services to conform to the study's eligibility 
Table 2 Primary and exploratory outcomes of the ImpleMentAll study

\begin{tabular}{|c|c|c|c|c|}
\hline & Outcome & Instrument & Organizational level & Staff level \\
\hline Primary outcome & Degree of normalization & NoMAD (20 items) & - & Baseline, 3-monthly \\
\hline \multirow[t]{8}{*}{ Exploratory outcome } & Demographics & Self-developed questionnaire & Once during the study period & Baseline \\
\hline & Service uptake & $\begin{array}{l}\text { Self-developed questionnaire } \\
\text { Data source: administrative data } \\
\text { basis (e.g. iCBT platform) }\end{array}$ & Baseline, 3-monthly & - \\
\hline & Implementation costs & $\begin{array}{l}\text { Self-developed questionnaire } \\
\text { Data source: financial administration }\end{array}$ & Baseline, 3-monthly & - \\
\hline & Exposure & Event-based platform log-files & Continuous & - \\
\hline & Satisfaction & CSQ (3 items) & End of exposure time & - \\
\hline & Usability & SUS (10 items) & End of exposure time & - \\
\hline & Perceived impact & Visual analogue scale (1 item) & End of exposure time & - \\
\hline & Organizational Readiness & ORIC (12 items) & - & Baseline, 3-monthly \\
\hline
\end{tabular}

Outcomes, assessment instruments, the level on which the outcomes are assessed, and measurements' time intervals. All staff-level questionnaires have been translated and adapted into local languages using a standardized translation guide

criteria. For the staff-level outcome, a series of simulation studies was conducted using a multi-level degree of normalization data (i.e. NoMAD items) to estimate the minimal required number of staff members to sufficiently power the analysis. Here, a 5\% increase in absolute normalization scores and an increased 3-month growth rate from .05 to .10 are assumed to be statistically decisive in superiority for either condition. The cluster sample size of 12 clusters, with 15 staff participants per implementation site per measurement wave, achieves $>80 \%$ power to detect this effect, using a twosided test with a significance level $\alpha$ of .05 . Taking a conservative study drop-out of $20 \%$ into account, the minimum staff sample size was set to $n=19$ per implementation site. In line with the closed cohort design, each participant will be measured continuously over a period of 10 measurement waves. For all 12 clusters, this results in a total minimum sample size of 228 staff participants with 2280 repeated data points.

\section{Randomization}

Implementation sites will be randomly allocated to one of six groups (two implementation sites per group, see Fig. 3) prior to the start of the study. Randomization will be conducted by a computerized random number generator using $\mathrm{R}$ [54]. No constraints to randomization will be applied. The allocation scheme can only be accessed by the central trial coordination team. Any other investigators and all study participants within the implementation sites will be blinded to the crossover sequence. Three months prior to cross-over, the two clusters randomized for rollout, the process evaluation team and the team involved in supportive activities of these sites, will be informed in order to prepare any organizational prerequisites necessary for using the ItFits-toolkit.

\section{Statistical methods}

For the normalization outcomes, a three-level GLMM will be conducted, with 'Wave' clustered at the 'Staff' level, and 'Staff' clustered at the 'Site' level, accounting for the correlation structure in the outcome. Random effects will be used to assess correlations between observations within and across units in the same clusters. Each regression parameter, including the intercept, will be allowed to vary within cluster levels 'Staff' and 'Site'. Efficiency of the implementation process (i.e. normalization and service uptake divided by implementation costs) will be included in the separate analyses.

It will be tested whether the introduction of the ItFitstoolkit influences iCBT service uptake by patients across and within sites $[55,56]$. An effect of the toolkit is demonstrated when service uptake shows a significant main effect of ItFits-toolkit use or an interaction effect of ItFits-toolkit use and measurement wave (time). Consequently, trial data will be analysed using generalized linear mixed modelling (GLMM) [55] with service uptake as the dependent variable, and measurement wave (time), ItFits-toolkit use (yes/ no), and interaction between time and ItFits-toolkit use as independent variables. To account for the expected intervention lag effect, a fractional term for the 'ItFits' parameter will be included in the 6-month minimal exposure time (ranging from 0 to 1 , i.e. $0-1 / 2-1$ ). Service uptake outcomes will be modelled in a two-level GLMM, since these measures are collected at the site level only. Thus, to account for the correlation structure of the uptake outcome, 'Wave' is modelled to be clustered at the 'Site' level. All regression parameters will be allowed to vary.

For exploratory purposes, measures of exposure to the ItFits-toolkit (event-based log files showing intensity of use and level of continuous use), CSQ, SUS, and ORIC questionnaire data will be added as additional predictors of outcome in the above-described regression models. 
In the analyses, all observed data will be included following the intention-to-treat principle. The ability of mixed models to estimate model parameters in the presence of missing observations will be used, and increased uncertainty caused by missing values will be accepted as a given quality of the results.

\section{Process evaluation}

\section{Implementation-as-usual}

The implementation-as-usual process evaluation will explore implementation-as-usual activities in which implementation sites were engaged in prior to receiving the ItFits-toolkit. This analysis will identify and describe these implementation actions and determinants they focused on.

\section{The ItFits-toolkit}

A qualitative process evaluation will be conducted to study how the effects of the ItFits-toolkit were achieved and to obtain a better understanding of the underlying theoretical and conceptual mechanisms of tailored implementation. The process evaluation will focus on (1) understanding what implementers do with the ItFitstoolkit, (2) understanding and describing how the ItFitstoolkit gets reconfigured and adapted within and across settings when it is used, and (3) identifying, describing, and understanding the micro-, meso-, and macromechanisms that shape ItFits-toolkit use within and between implementation sites. The ItFits-toolkit process evaluation will be theoretically informed by Normalization Process Theory (NPT) [35], Selfdetermination Theory (SDT) [57], and work within organization studies, especially on organizational routines $[58,59]$. The conceptual ideas within these bodies of literature will enable the research team to focus on the work through which the ItFits-toolkit is implemented, what motivates individuals to work with the ItFits-toolkit, and how the ItFits-toolkit facilitates the structuring of time, resources, and people to support implementation. Four qualitative research methods will be used within the process evaluation, including theoryinformed interviews with Implementation Leads and main informants (e.g. implementation practitioners and trainers of the ItFits-toolkit), in situ and distal observations of implementers engaged with the ItFits-toolkit, process data from the use of the ItFits-toolkit, and analysis of documents, texts, and technological specifications produced and made available by ItFits-toolkit users. Interviews with the Implementation Leads will be generally conducted in English. More focused qualitative observations will be conducted in some purposively sampled sites according to the spoken languages of the researchers. This work will involve in situ and distil observations of meetings of the core implementation teams and meetings with key stakeholders. Qualitative data will be analysed according to the analytical framework developed and will be conducted according to the standard procedures of rigorous qualitative analysis [60]. Analysis will occur concurrently with data collection following the stepped order of implementation sites' entry into the trial. This allows for emerging trends found in earlier rounds of fieldwork to be explored in subsequent ones. All data will be audio-recorded, transcribed verbatim, and analysed using framework analysis [61]. The results of the ItFits-toolkit process evaluation will be used to further inform the outcome evaluation.

\section{Discussion}

The ImpleMentAll study is a large-scale international collaborative research project designed to study the effectiveness of tailored implementation and better understand the mechanisms of implementing iCBT for common mental disorders. A newly developed digitally accessible toolkit by which implementation strategies are prospectively developed, adapted, applied, and evaluated will be tested for its effectiveness compared to usual implementation activities. The toolkit will be introduced in twelve different mental health care organizations in nine countries across Europe and Australia. This real-world research setting provides a variety in health care systems, iCBT services, policies, implementation climate, and levels of experience in delivering iCBT. Tailored implementation is thought to be generically applicable across care contexts. The conceptual idea behind the tailoring process builds on recent literature findings and methods in the field of implementation (publication forthcoming).

The ImpleMentAll project applies a stepped wedge cluster randomized controlled trial design to determine the effectiveness of the ItFits-toolkit. Reasons for choosing the SWT design include practical feasibility and flexibility, fairness, and strength of the evidence [56]. A classical randomized controlled trial would not have been feasible due to the highly heterogeneous and reasonably small sample of organizations included in the study. It would not have been possible to alternatively randomize participants at individual level as this would have conflicted the naturalistic setting of the study. Due to repeated measures, a SWT requires less participants to adequately power the statistical analysis. However, achieving and maintaining a stable sample for the duration of the study will be challenging. Biases due to time trends such as organizational restructuring, data regulation policies and legislation, and technological advances need to be considered given the potential large intervention lag effect in implementation trials. A SWT design allows for the possibility to adjust for time trends in outcomes. It distributes the chances of such time trends affecting the dependent variables equally across the 
participating sites. Furthermore, the design increases practical feasibility of the study as it allows for sequential, batch-wise training of the implementation sites to the ItFits-toolkit and keeps guidance limited to those groups who are in the exposure condition.

The use of such a trial design is novel, but the literature in the field is growing at the time of writing. Generic guidance $[33,62]$ on how to conduct a SWT is available. However, there is no scientific literature on particular components of the design applied to implementation research, most notably the minimal exposure time to exert an effect in relation to the potential intervention lag effect. A minimal exposure period of 6 months was chosen as it strikes a reasonable balance between time constraints of the total trial period and to constitute to meaningful exposure by finishing one complete cycle of the ItFits-toolkit. Carry-over effects might occur when implementation sites cross over from the control to the experimental condition. The process evaluation conducted within this project is expected to shed light on the implementation mechanisms considering potential carry-over effects. This will be regarded when interpreting the results of the effectiveness study.

The ImpleMentAll study does not include an evaluation of the clinical effectiveness of the iCBT services per se, as the focus lies on establishing the effectiveness of the ItFits-toolkit-the implementation intervention. The effectiveness of the ItFits-toolkit will be investigated by measuring implementation outcomes mainly on organization and staff level, anticipating that change processes to successfully implement iCBT in routine care are predominantly taking place at those levels. The perspective of the patient using the $\mathrm{iCBT}$ services will be considered indirectly by assessing the completion rates of the services by the patients. This means that the implementation could be perceived as successful from the perspective of the organization and staff, irrespective of improvements in clinical outcomes (e.g. symptom reduction) in the patient.

The field of implementation research is still young, and therefore, access to thoroughly validated and theory-based measurement instruments is limited [63, 64]. The measurement instruments used to assess implementation success or related outcomes (i.e. NoMAD, ORIC, and implementation costs measure) are relatively new, though promising [42-44, 51]. Experience of the field to use these validated measures is low, and therefore, the interpretation might not be as straightforward compared to wellestablished instruments. Uncertainties around sensitivity to change in these instruments are present, and further psychometric validation is planned.

The ImpleMentAll study engages in a number of challenges, mostly related to the relatively new concepts under study, the fast-changing world of technology- assisted interventions, and the complex and heterogenous implementation contexts. In that sense, the ImpleMentAll study is well-positioned to take the first step towards exploring the effectiveness of an online self-help toolkit for a tailored implementation supporting the implementation of evidence-based eHealth in mental health care. As such, it will contribute to implementation science by investigating the effectiveness of tailored implementation and providing a better understanding of the process and methods for tailoring implementation strategies. Measurement instruments for implementation outcomes related to implementation success will be further improved and validated. If effective, the ItFits-toolkit will be made available to implementers supporting them in identifying barriers, selecting, localizing, and applying appropriate implementation strategies for successfully implementing $\mathrm{iCBT}$ in their practices. This will ultimately be beneficial for the large proportion of individuals in need of evidence-based health care.

\section{Trial registration}

This protocol was registered with ClinicalTrials.gov on August 29, 2018 (No. NCT03652883). Results of the study will be reported to ClinicalTrials.gov. The research team will monitor protocol compliance and record the progress of the study. The principal investigator will submit annual reports on study progress to the European Commission, the main funder of the project.

\section{Trial status}

The ImpleMentAll study runs from March 2018 to March 2021. Implementation sites across Europe and Australia were recruited following a purposeful sampling approach. According to the closed cohort design of the study, recruitment of clusters (implementation sites) was completed prior to the first measurement wave (before September 2018). Subsequently, the included implementation sites engaged in recruiting a minimum number of staff participants ( $n=19$ per cluster) to ensure a stable and sufficient sample for repeated data collection during the trial. Staff participant recruitment is open and continues throughout the trial to allow for replacements of potential study drop-outs. Implementation sites have localized the study protocol, translated measurement instruments and obtained ethical approval. Ethical approval for the process evaluation was granted by the University of Northumbria, UK. The randomization scheme has been finalized. Data collection will be completed in late 2020 and the first results are expected to become available in 2021 .

\section{Abbreviations}

CBT: Cognitive Behavioural Therapy; CSQ: Client Satisfaction Questionnaire; GLMM: Generalized Linear Mixed Modelling; iCBT: Internet-delivered 
Cognitive Behavioural Therapy; ItFits-toolkit: Integrated Theory-based Framework for Implementation Tailoring Strategies; NoMAD: Normalization Measure Development Questionnaire; NPT: Normalization process theory; ORIC: Organizational Readiness for Implementing Change; SDT: Selfdetermination Theory; SUS: System Usability Scale; SWT: Stepped wedge cluster randomized controlled trial

\section{Acknowledgements}

The authors want to express their gratitude to the funding bodies, the European Union by means of the European Commission's Horizon 2020 research programme and the Australian government, for being provided with the financial capacity to conduct the ImpleMentAll study.

We thank the ImpleMentAll consortium for every individual contribution and team effort and for bringing together the required expertise, experience, and organizational capacity to make this study a success. Specifically, we thank the local research and implementation teams, the internal Scientific Steering Committee, and the External Advisory Board for their input in designing the ImpleMentAll study.

We remember Dr. Jeroen Ruwaard. His unexpected death (16 July 2019)

leaves behind an inexpressible void, to his family, his friends, close colleagues, the ImpleMentAll consortium, and the wider scientific and mental health community. Jeroen's involvement with the study and mentorship was indispensable and essential; from drafting the first ideas for the grant application and writing the full proposal to providing the methodological and statistical foundations for the stepped wedge trial design, the development of the ItFits-toolkit, trial data management, and ethics. Science and data for the benefit of mental health care were Jeroen's passion, and he acted as the senior methodologist of the study: better one number well defined than a bucket full of noise. We want to express our gratitude towards Jeroen for the effort he made in realizing this study.

\section{ImpleMentAll consortium:}

Adriaan Hoogendoorn

Alison Calear

Andia Meksi

Anna Sofie Rømer

Anne Etzelmüller

Antoine Yrondi

Arlinda Cerga-Pashoja

Besnik Loshaj

Bridianne O'Dea

Bruno Aouizerate

Camilla Stryhn

Carl May

Carmen Ceinos

Caroline Oehler

Catherine Pope

Christiaan Vis

Christine Marking

Claire van Genugten

Claus Duedal Pedersen

Corinna Gumbmann

Dana Menist

David Daniel Ebert

Denise Hanssen

Elena Heber

Els Dozeman

Emilie Nielsen

Emmanuel Haffen

Enrico Zanalda

Erida Nelaj

Erik Van der Eycken

Eva Fris

Fiona Shand

Gentiana Qirjako

Géraldine Visentin

Heleen Riper

Helen Christensen

Ingrid Titzler

Isabel Weber

Isabel Zbukvic

Jeroen Ruwaard
Jerome Holtzmann

Johanna Freund

Johannes H Smit

Jordi Piera-Jiménez

Josep Penya

Josephine Kreutzer

Josien Schuurmans

Judith Rosmalen

Juliane Hug

Kim Mathiasen

Kristian Kidholm

Kristine Tarp

Leah Bührmann

Linda Lisberg

Ludovic Samalin

Maite Arrillaga

Margot Fleuren

Maria Chovet

Marion Leboyeer

Martine Pool

Mette Atipei Craggs

Mette Maria Skjøth

Naim Fanaj

Nicole Cockayne

Philip J. Batterham

Pia Driessen

Pierre Michel Llorca

Rhonda Wilson

Ricardo Araya

Robin Kok

Sebastian Potthoff

Sergi García Redondo

Sevim Mustafa

Tim Rapley

Tracy Finch

Ulrich Hegerl

Virginie Tsilibaris

Wissam Elhage

Ylenia Sacco

Monitored email address for group correspondence: l.buhrmann@vu.nl

\section{Authors' contributions}

$C V$, JS, JR, MF, AE, JP, TF, TR, CM, CP, JHS, HR, and LB designed the study. CDP was the project coordinator of ImpleMentAll from 2017 to 2020, followed by KM in 2020 until today; CV is the scientific coordinator. DDE, UH, CDP, CM, JHS, and HR are part of the Scientific Steering Committee. CVG, JP, $D D E, I T, U H, C O, P B, A C, H C, I Z, J R, D H, E Z, Y S, B A, L S, K T, K M, N F, S M, G Q$, and $A C P$ represent the local implementation sites and function as research leads for the ImpleMentAll partners. EvdE represents the patient perspective to the project. JS, TF, JP, JH, AE, MMS, CDP, and CV lead the work packages within the ImpleMentAll study. JS, JR, and CVG build the central trial management team. TR, TF, SP, CM, CP, JP, CV, and LB developed and tested the ItFits-toolkit. CV and LB provided the introductory training and technical support for the ItFits-toolkit use together with JP. TF, TR, and SP lead the ItFits-toolkit process evaluation. AE leads the work around the implementation-as-usual process evaluation. All authors provided feedback and suggestions for this manuscript and agreed on the publication. The authors read and approved the final manuscript.

\section{Funding}

This project is funded by the European Union's Horizon 2020 research and innovation programme under grant agreement No. 733025 and receives funding from the NHMRC-EU programme by the Australian Government (1142363). Funding bodies had no influence on the design of this study.

\section{Availability of data and materials}

The translations of the measurement instruments NoMAD and ORIC are available for public use and can be accessed here https://www.implementall. eu/9-outcomes-and-resources.html. 


\section{Ethics approval and consent to participate}

Implementation sites have presented the study protocol for the implementation effectiveness study to local medical ethical committees and relating regulatory agencies. The committees concluded that this study is to be regarded as implementing evidence-based interventions and no human or animal experiments are involved. Approval letters from the committees are available on request. In Albania, there is no functional national ethical committee yet. As soon as this committee is established, the Albanian partner will submit their protocol for approval. In the meantime, they received approval from their institution to participate in the trial. Ethical approval for the process evaluation was granted by the University of Northumbria, UK (Submission Ref: 11039). Participants are required to provide and sign an informed consent indicating the purpose of the study, the nature, use and management of their data.

\section{Consent for publication}

Not applicable.

\section{Competing interests}

Professor Bruno Aouizerate has received honoraria or consulting fees from Janssen-Cilag, Lundbeck, and Lilly. Dr. Samalin has received grants, honoraria, or consulting fees from Janssen-Cilag, Lundbeck, and Otsuka. All other authors declare that they have no competing interests.

\section{Author details}

'Department of Clinical, Neuro-, \& Developmental Psychology, Faculty of Behavioural and Movement Sciences, VU Amsterdam, Amsterdam, The Netherlands. ${ }^{2}$ Amsterdam UMC, Vrije Universiteit Amsterdam, Psychiatry, Amsterdam Public Health Research Institute, Amsterdam, The Netherlands. ${ }^{3}$ Department of Research and Innovation, GGZ inGeest Specialized Mental Health Care, Amsterdam, The Netherlands. ${ }^{4}$ Dutch Nurses Association, Utrecht, The Netherlands. ${ }^{5}$ GET.ON Institute, Hamburg, Germany. ${ }^{6}$ Department of Research and Innovation, Badalona Serveis Assistencials, Badalona, Spain. ${ }^{7}$ Department of Nursing, Midwifery and Health, Northumbria University, Newcastle upon Tyne, UK. ${ }^{8}$ Department of Social Work, Education and Community Wellbeing, Northumbria University, Newcastle upon Tyne, UK. ${ }^{9}$ Faculty of Health and Life Sciences, Northumbria University, Newcastle upon Tyne, UK. ${ }^{10}$ Fondation FondaMental, Creteil, France. ${ }^{11}$ Regional Reference Center for the Management and Treatment of Anxiety and Depressive Disorders, Expert Center for Treatment-Resistant Depression, $\mathrm{CH}$ Charles Perrens, University of Bordeaux, Bordeaux, France. ${ }^{12}$ Centre for Mental Health Research, Research School of Population Health, The Australian National University, Canberra, Australia. ${ }^{13}$ Black Dog Institute, University of New South Wales, Randwick, Australia. ${ }^{14}$ Centre for Innovative Medical Technology, Odense University Hospital, Odense, Denmark. ${ }^{15}$ Department of Clinical Psychology and Psychotherapy, Friedrich-Alexander University of Erlangen-Nuremberg, Erlangen, Germany. ${ }^{16}$ GAMIAN Europe, Brussels, Belgium. ${ }^{17}$ Mental Health Center Prizren, Prizren, Kosovo. ${ }^{18}$ College of Medical Sciences Rezonanca, Prishtina, Kosovo. ${ }^{19}$ University Medical Center Groningen, Interdisciplinary Center for Psychopathology and Emotion Regulation, University of Groningen, Groningen, The Netherlands. ${ }^{20}$ Department of Psychiatry, Psychosomatics and Psychotherapy, Goethe-Universität Frankfurt, Frankfurt, Germany. ${ }^{21}$ European Alliance Against Depression e.V., Leipzig, Germany. ${ }^{22}$ Research Unit for Depression and Anxiety, Aarhus University Hospital, Aarhus N, Denmark. ${ }^{23}$ Faculty of Public Health and Policy, London School of Hygiene and Tropical Medicine, London, UK. ${ }^{24}$ Faculty of Education, University St. Kliment Ohridski, Bitola, North Macedonia. ${ }^{25}$ Stiftung Deutsche Depressionshilfe, Leipzig, Germany. ${ }^{26}$ Department of Population Health, London School of Hygiene \& Tropical Medicine, London, UK. ${ }^{27}$ Nuffield Department of Primary Care Health Sciences, University of Oxford, Oxford, UK. ${ }^{28}$ Department of Public Health, Faculty of Medicine, University of Medicine, Tirana, Albania. ${ }^{29}$ Dipartimento di Salute Mentale, Azienda Sanitaria Locale Torino 3, Turin, Italy. ${ }^{30}$ Department of Psychiatry, CHU Clermont-Ferrand, University of Clermont Auvergne, Clermont-Ferrand, France. ${ }^{31}$ Danish Centre for Health Economics, Department of Public Health, University of Southern Denmark, Odense, Denmark. ${ }^{32}$ Centre for Telepsychiatry, Region of Southern Denmark, Denmark. ${ }^{33}$ Orygen, Parkville, Victoria, Australia. ${ }^{34}$ Centre for Youth Mental Health, The University of Melbourne, Parkville, Victoria, Australia.
Received: 3 February 2020 Accepted: 14 August 2020

Published online: 28 October 2020

\section{References}

1. Kessler RC, Aguilar-Gaxiola S, Alonso J, Chatterji S, Lee S, Ormel J, et al. The global burden of mental disorders: an update from the WHO World Mental Health (WMH) surveys. Epidemiol Psychiatr Sci. 2009;18(1):23-33.

2. Ebert DD, Van Daele T, Nordgreen T, Karekla M, Compare A, Zarbo C, Kaehlke F. Internet and mobile-based psychological interventions: Applications, efficacy and potential for improving mental health. A report of the EFPA E-Health Taskforce (vol 23, pg 167, 2018). Eur Psychol. 2018;23(3): 269.

3. Josephine K, Josefine L, Philipp D, David E, Harald B. Internet- and mobilebased depression interventions for people with diagnosed depression: a systematic review and meta-analysis. J Affect Disord. 2017;223:28-40 Available from: https://www.sciencedirect.com/science/article/pii/S0165032 717307255. Cited 2019 Nov 6.

4. Lindefors N, Andersson G, editors. Guided Internet-based treatments in psychiatry. Cham: Springer International Publishing; 2016.

5. Karyotaki E, Riper H, Twisk J, Hoogendoorn A, Kleiboer A, Mira A, et al. Efficacy of self-guided internet-based cognitive behavioral therapy in the treatment of depressive symptoms a meta-analysis of individual participant data. JAMA Psychiatry. 2017;74(4):351-9 Available from: https:/jamanetwork. com/journals/jamapsychiatry/article-abstract/2604310. Cited 2019 Jul 17.

6. Karyotaki E, Ebert DD, Donkin L, Riper H, Twisk J, Burger S, et al. Do guided internet-based interventions result in clinically relevant changes for patients with depression? An individual participant data meta-analysis. Clin Psychol Rev. 2018;63:80-92.

7. Carlbring P, Andersson G, Cuijpers P, Riper H, Hedman-Lagerlöf E. Internetbased vs. face-to-face cognitive behavior therapy for psychiatric and somatic disorders: an updated systematic review and meta-analysis. Cogn Behav Ther. 2018;47(1):1-18.

8. Kooistra LC, Wiersma JE, Ruwaard J, Neijenhuijs K, Lokkerbol J, van Oppen P, et al. Cost and effectiveness of blended versus standard cognitive behavioral therapy for outpatients with depression in routine specialized mental health care: pilot randomized controlled trial. J Med Internet Res. 2019;21(10):e14261 Available from: http://www.jmir.org/2019/10/e14261/.

9. Byrnes A, Young A, Mudge A, Banks M, Clark D, Bauer J. Prospective application of an implementation framework to improve postoperative nutrition care processes: evaluation of a mixed methods implementation study. Nutr Diet. 2018;75(4):353-62 Available from: http://doi.wiley.com/1 0.1111/1747-0080.12464. Cited 2019 Jul 17.

10. Eccles MP, Armstrong D, Baker R, et al. An implementation research agenda. Implementation Sci. 2009;4:18 https://doi.org/10.1186/1748-5908-4-18.

11. Greenhalgh T, Robert G, Macfarlane F, Bate P, Kyriakidou O. Diffusion of innovations in service organizations: systematic review and recommendations. Milbank Q. 2004;82(4):581-629.

12. May C. Towards a general theory of implementation. Implement Sci. 2013; 8(1):18.

13. Stelk WJ. Implementing health-care innovations: in search of a theoretical foundation for a science of implementation. Int J Ment Health. 2006;35(2): 35-49 Available from: https://www.tandfonline.com/doi/full/10.2753/IMH002 0-7411350203. Cited 2019 Jul 17.

14. Emmelkamp PMG, David D, Beckers T, Muris P, Cuijpers P, Lutz W, et al. Advancing psychotherapy and evidence-based psychological interventions. Int J Methods Psychiatr Res. 2014;23(S1):58-91 Available from: http://doi. wiley.com/10.1002/mpr.1411. Cited 2019 Jul 17.

15. Hadjistavropoulos HD, Nugent MM, Dirkse D, Pugh N. Implementation of internet-delivered cognitive behavior therapy within community mental health clinics: a process evaluation using the consolidated framework for implementation research. BMC Psychiatry. 2017;17(1):331 Available from: http://bmcpsychiatry.biomedcentral.com/articles/10.1186/s12888-017-1496-7. Cited 2019 Jul 17.

16. Kazdin AE, Blase SL. Rebooting psychotherapy research and practice to reduce the burden of mental illness. Perspect Psychol Sci. 2011;6(1):21-37 Available from: http://journals.sagepub.com/doi/10.1177/1745691610393527. Cited 2019 Jul 17.

17. Rabin BA, Glasgow RE. Dissemination and implementation of eHealth interventions. In: eHealth applications: promising strategies for behavior change; 2012. p. 221-45. 
18. McHugh RK, Barlow DH. The dissemination and implementation of evidence-based psychological treatments: a review of current efforts. In: The neurotic paradox: progress in understanding and treating anxiety and related disorders; 2018. p. 409-32. Available from: https://psycnet.apa.org/ record/2010-02208-010. Cited 2019 Jul 17.

19. Grol R, Grimshaw J. Evidence-based implementation of evidence-based medicine. Jt Comm J Qual Improv. 1999;25(10):503-13.

20. Folker AP, Mathiasen K, Lauridsen SM, Stenderup E, Dozeman E, Folker MP. Implementing internet-delivered cognitive behavior therapy for common mental health disorders: a comparative case study of implementation challenges perceived by therapists and managers in five European internet services. Internet Interv. 2018;11:60-70 Available from: https://www. sciencedirect.com/science/article/pii/S2214782917301203. Cited 2019 Jul 17.

21. Titzler I, Saruhanjan K, Berking M, Riper H, Ebert DD. Barriers and facilitators for the implementation of blended psychotherapy for depression: a qualitative pilot study of therapists' perspective. Internet Interv. 2018;12:15064 Available from: https://www.sciencedirect.com/science/article/pii/S2214 782917300908. Cited 2019 Jul 17.

22. Wilhelmsen M, Høifødt RS, Kolstrup N, Waterloo K, Eisemann M, Chenhall R, et al. Norwegian general practitioners'perspectives on implementation of a guided web-based cognitive behavioral therapy for depression: a qualitative study. J Med Internet Res. 2014;16(9) Available from: https://www.jmir.org/ article/view/3556/1. Cited 2019 Jul 17.

23. Vis C, Mol M, Kleiboer A, Bührmann L, Finch T, Smit J, et al. Improving implementation of eMental health for mood disorders in routine practice: systematic review of barriers and facilitating factors. J Med Internet Res. 2018;20 Available from: https://mental.jmir.org/2018/1/e20 Cited 2019 Jul 17

24. May CR, Johnson M, Finch T. Implementation, context and complexity. Implement Sci. 2016;11(1):141 Available from: http://implementationscience. biomedcentral.com/articles/10.1186/s13012-016-0506-3. Cited 2019 Jul 17.

25. Powell BJ, McMillen JC, Proctor EK, Carpenter CR, Griffey RT, Bunger AC, et al. A compilation of strategies for implementing clinical innovations in health and mental health. Med Care Res Rev. 2012;69(2):123-57 Available from: http://journals.sagepub.com/doi/10.1177/1077558711430690. Cited 2019 Jul 17.

26. Powell BJ, Proctor EK, Glass JE. A systematic review of strategies for implementing empirically supported mental health interventions. Res Soc Work Pract. 2014;24(2):192-212 Available from: http://journals.sagepub.com/ doi/10.1177/1049731513505778. Cited 2019 Jul 17.

27. Baker R, Camosso-Stefinovic J, Gillies C, Shaw EJ, Cheater F, Flottorp S, et al. Tailored interventions to address determinants of practice. Cochrane Database Syst Rev. 2015; Available from: http://doi.wiley.com/10.1002/14651 858.CD005470.pub3. Cited 2019 Jul 17.

28. Wensing M. The Tailored Implementation in Chronic Diseases (TICD) project: introduction and main findings. Implement Sci. 2017;12(1):5 Available from: http://implementationscience.biomedcentral.com/articles/10.1186/s13012016-0536-x. Cited 2019 Jul 17.

29. Aarons GA, Ehrhart MG, Farahnak LR, Sklar M. Aligning leadership across systems and organizations to develop a strategic climate for evidencebased practice implementation. Annu Rev Public Health. 2014;35:255-74.

30. Wensing M, Oxman A, Baker R, Godycki-Cwirko M, Flottorp S, Szecsenyi J, et al. Tailored Implementation for Chronic Diseases (TICD): a project protocol. Implement Sci. 2011;6(1):103 Available from: http://implementationscience. biomedcentral.com/articles/10.1186/1748-5908-6-103. Cited 2019 Jul 17.

31. Krause J, Van Lieshout J, Klomp R, Huntink E, Aakhus E, Flottorp S, et al. Identifying determinants of care for tailoring implementation in chronic diseases: an evaluation of different methods. Implement Sci. 2014;9(1):102 Available from: http://implementationscience.biomedcentral.com/articles/1 0.1186/s13012-014-0102-3. Cited 2019 Jul 17.

32. Ruwaard J, Kok R. Wild West eHealth: time to hold our horses? Eur Health Psychol. 2015;17(1):45-9.

33. Hemming K, Taljaard M, McKenzie JE, Hooper R, Copas A, Thompson JA, et al. Reporting of stepped wedge cluster randomised trials: extension of the CONSORT 2010 statement with explanation and elaboration. BMJ. 2018;363: k1614 Available from: https://www.bmj.com/content/363/bmj.k1614/rapidresponses?utm_campaign=tbmj\&utm_medium $=$ cpc\&utm_source= trendmd\&utm_term=usage-042019\&utm_content=consumer. Cited 2019 Jul 17.

34. Dobson D, Dobson K. Evidence-based practice of cognitive-behavioral therapy; 2018
35. May C, Finch T. Implementing, embedding, and integrating practices: an outline of normalization process theory. Sociology. 2009;43(3):535-54 Available from: http://journals.sagepub.com/doi/10.1177/0038038509103208. Cited 2019 Jul 17.

36. May C. Agency and implementation: understanding the embedding of healthcare innovations in practice. Soc Sci Med. 2013;78(1):26-33 Available from: https://www.sciencedirect.com/science/article/pii/S0277953612007708. Cited 2019 Jul 17.

37. Mair FS, May C, O'Donnell C, Finch T, Sullivan F, Murray E. Factors that promote or inhibit the implementation of e-health systems: an explanatory systematic review. Bull World Health Organ. 2012;90(5):357-64 Available from: https://www.scielosp.org/scielo.php?pid=S0042-96862012000500011 \&script=sci_arttext\&tlng=es. Cited 2019 Jul 17.

38. Ross J, Stevenson F, Lau R, Murray E. Exploring the challenges of implementing e-health: a protocol for an update of a systematic review of reviews. BMJ Open. 2015;5 https://doi.org/10.1136/bmjopen-2014-006773.

39. Powell BJ, Waltz TJ, Chinman MJ, Damschroder LJ, Smith JL, Matthieu MM et al. A refined compilation of implementation strategies: results from the Expert Recommendations for Implementing Change (ERIC) project. Implement Sci. 2015;10(1):21.

40. Hoffmann TC, Glasziou PP, Boutron I, Milne R, Perera R, Moher D, et al. Better reporting of interventions: Template for Intervention Description and Replication (TIDieR) checklist and guide. BMJ. 2014;348:g1687.

41. Rapley T, Girling M, Mair FS, Murray E, Treweek S, McColl E, et al. Improving the normalization of complex interventions: part 1 - development of the NoMAD instrument for assessing implementation work based on normalization process theory (NPT). BMC Med Res Methodol. 2018;18(1):133 Available from: https://bmcmedresmethodol.biomedcentral.com/articles/1 0.1186/s12874-018-0590-y. Cited 2019 Jul 17.

42. Finch TL, Girling M, May CR, Mair FS, Murray E, Treweek S, et al. Improving the normalization of complex interventions: part 2 - validation of the NoMAD instrument for assessing implementation work based on normalization process theory (NPT). BMC Med Res Methodol. 2018;18(1):135 Available from: https://bmcmedresmethodol.biomedcentral.com/articles/1 0.1186/s12874-018-0591-x. Cited 2019 Jul 17

43. Vis C, Ruwaard J, Finch T, Rapley T, de Beurs D, van Stel H, et al. Toward an objective assessment of implementation processes for innovations in health care: psychometric evaluation of the Normalization Measure Development (NoMAD) questionnaire among mental health care professionals. J Med Internet Res. 2019;21(2):e12376.

44. Elf M, Nordmark S, Lyhagen J, Lindberg I, Finch T, Åberg AC. The Swedish version of the Normalization Process Theory Measure S-NoMAD: translation, adaptation, and pilot testing. Implement Sci. 2018;13(1):146 Available from: https://implementationscience.biomedcentral.com/articles/10.1186/s13012018-0835-5. Cited 2019 Jul 17.

45. Larsen DL, Attkisson CC, Hargreaves WA, Nguyen TD. Assessment of client/ patient satisfaction: development of a general scale. Eval Program Plann. 1979;2(3):197-207 Available from: https://www.sciencedirect.com/science/ article/pii/0149718979900946. Cited 2019 Jul 17.

46. Attkisson CC, Zwick R. The client satisfaction questionnaire. Psychometric properties and correlations with service utilization and psychotherapy outcome. Eval Program Plann. 1982;5(3):233-7 Available from: https://www. sciencedirect.com/science/article/pii/014971898290074X. Cited 2019 Jul 17.

47. Boß L, Lehr D, Reis D, Vis C, Riper H, Berking M, et al. Reliability and validity of assessing user satisfaction with web-based health interventions. J Med Internet Res. 2016;18(8):e234.

48. Brooke J. SUS-A quick and dirty usability scale. Usability Eval Ind. 1996; 189(194):4-7.

49. Lewis JR, Sauro J. The factor structure of the system usability scale. In: Lecture notes in computer science (including subseries Lecture notes in artificial intelligence and lecture notes in bioinformatics); 2009. p. 94-103. Available from: https://link.springer.com/chapter/10.1007/978-3-642-028069_12. Cited 2019 Jul 17.

50. Rubenstein LV, Danz MS, Crain AL, Glasgow RE, Whitebird RR, Solberg LI. Assessing organizational readiness for depression care quality improvement: relative commitment and implementation capability. Implement Sci. 2014; 9(1):173 Available from: http://implementationscience.biomedcentral.com/ articles/10.1186/s13012-014-0173-1. Cited 2019 Jul 17.

51. Shea CM, Jacobs SR, Esserman DA, Bruce K, Weiner BJ. Organizational readiness for implementing change: a psychometric assessment of a new measure. Implement Sci. 2014;9(1):7 Available from: http:// 
implementationscience.biomedcentral.com/articles/10.1186/1748-5908-9-7. Cited 2019 Jul 17.

52. Weiner BJ. A theory of organizational readiness for change. Implement Sci. 2009:4(1):67 Available from: http://implementationscience.biomedcentral. com/articles/10.1186/1748-5908-4-67. Cited 2019 Jul 17.

53. The ImpleMentAll Project. Available from: https://www.implementall.eu/9outcomes-and-resources.htm|\#NoMADtranslations. Cited 2019 Oct 28.

54. Uschner D, Schindler D, Hilgers R-D, Heussen N. randomizeR: an R package for the assessment and implementation of randomization in clinical trials. J Stat Softw. 2018;85(8):1-22.

55. Hussey MA, Hughes JP. Design and analysis of stepped wedge cluster randomized trials. Contemp Clin Trials. 2007;28(2):182-91 Available from: https://www.sciencedirect.com/science/article/pii/S1551714406000632. Cited 2019 Jul 17.

56. Hemming K, Haines TP, Chilton PJ, Girling AJ, Lifford RJ. The stepped wedge cluster randomised trial: rationale, design, analysis, and reporting. BMJ. 2015;350 Available from: https:/uww.bmj.com/content/350/bmj.h391.long. Cited 2019 Jul 17.

57. Ryan RM, Deci EL. Self-determination theory and the facilitation of intrinsic motivation, social development, and well-being. Am Psychol. 2000;55(1):68.

58. Becker MC. The concept of routines: some clarifications. Cambridge J Econ. 2005;29(2):249-62.

59. Greenhalgh T. Role of routines in collaborative work in healthcare organisations. BMJ. 2008;337:a2448.

60. Rapley T. Some pragmatics of data analysis. In: Silverman D, editor. Qualitative research. 4th ed. London: Sage; 2016.

61. Ritchie J, Lewis J. Qualitative research practice: a guide for social science students and researchers. London: Sage; 2003.

62. Copas AJ, Lewis JJ, Thompson JA, Davey C, Baio G, Hargreaves JR. Designing a stepped wedge trial: three main designs, carry-over effects and randomisation approaches. Trials. 2015;16(1):352 Available from: https:// trialsjournal.biomedcentral.com/articles/10.1186/s13063-015-0842-7. Cited 2019 Jul 17.

63. Lewis CC, Fischer S, Weiner BJ, Stanick C, Kim M, Martinez RG. Outcomes for implementation science: an enhanced systematic review of instruments using evidence-based rating criteria. Implement Sci. 2015;10(1):155 Available from: http://implementationscience.biomedcentral.com/articles/10.1186/s13 012-015-0342-x. Cited 2019 Jul 17.

64. Martinez RG, Lewis CC, Weiner BJ. Instrumentation issues in implementation science. Implement Sci. 2014;9(1):118 Available from: http:// implementationscience.biomedcentral.com/articles/10.1186/s13012-014-011 8-8. Cited 2019 Jul 17.

\section{Publisher's Note}

Springer Nature remains neutral with regard to jurisdictional claims in published maps and institutional affiliations.

Ready to submit your research? Choose BMC and benefit from:

- fast, convenient online submission

- thorough peer review by experienced researchers in your field

- rapid publication on acceptance

- support for research data, including large and complex data types

- gold Open Access which fosters wider collaboration and increased citations

- maximum visibility for your research: over $100 \mathrm{M}$ website views per year

At BMC, research is always in progress.

Learn more biomedcentral.com/submissions 